OPEN ACCESS

Edited by:

Sian M. Henson,

Queen Mary University of London,

United Kingdom

Reviewed by:

Natalie E. Riddell,

University of Surrey,

United Kingdom

Mathieu-Benoit Voisin,

Queen Mary University of London,

United Kingdom

*Correspondence:

Myriam Chimen

m.chimen@bham.ac.uk

Specialty section: This article was submitted to

T Cell Biology,

a section of the journal

Frontiers in Immunology

Received: 27 February 2021 Accepted: 13 April 2021

Published: 14 May 2021

Citation:

Nathan P, Gibbs JE, Rainger GE and Chimen $M$ (2021) Changes in

Circadian Rhythms Dysregulate Inflammation in Ageing: Focus on

Leukocyte Trafficking.

Front. Immunol. 12:673405. doi: 10.3389/fimmu.2021.673405

\section{Changes in Circadian Rhythms Dysregulate Inflammation in Ageing: Focus on Leukocyte Trafficking}

\author{
Poppy Nathan ${ }^{1}$, Julie Elizabeth Gibbs ${ }^{2}$, G. Ed Rainger ${ }^{3}$ and Myriam Chimen ${ }^{1 *}$ \\ ${ }^{1}$ MRC-Versus Arthritis Centre for Musculoskeletal Ageing Research, Institute of Inflammation and Ageing, College of Medical \\ and Dental Sciences, University of Birmingham, Birmingham, United Kingdom, ${ }^{2}$ Centre for Biological Timing, Faculty of \\ Biology Medicine and Health, University of Manchester, Manchester, United Kingdom, ${ }^{3}$ Institute of Cardiovascular Sciences, \\ College of Medical and Dental Sciences, University of Birmingham, Birmingham, United Kingdom
}

Leukocyte trafficking shows strong diurnal rhythmicity and is tightly regulated by circadian rhythms. As we age, leukocyte trafficking becomes dysregulated, contributing to the increased systemic, low-grade, chronic inflammation observed in older adults. Ageing is also associated with diminished circadian outputs and a dysregulation of the circadian rhythm. Despite this, there is little evidence to show the direct impact of age-associated dampening of circadian rhythms on the dysregulation of leukocyte trafficking. Here, we review the core mammalian circadian clock machinery and discuss the changes that occur in this biological system in ageing. In particular, we focus on the changes that occur to leukocyte trafficking rhythmicity with increasing age and consider how this impacts inflammation and the development of immune-mediated inflammatory disorders (IMIDs). We aim to encourage future ageing biology research to include a circadian approach in order to fully elucidate whether age-related circadian changes occur as a by-product of healthy ageing, or if they play a significant role in the development of IMIDs.

Keywords: inflammation, circadian rhythm, leukocytes, trafficking, chronotherapy

\section{INTRODUCTION}

Under homeostatic conditions, leukocytes migrate between the vasculature and different tissues for immune surveillance. In response to infections or injuries, leukocytes are recruited to the site of inflammation and play key roles in pathogen clearance and tissue repair. Temporal expression of adhesion molecules on the leukocyte surface and on endothelial cells (ECs) mediates leukocyte trafficking, in a process known as the leukocyte adhesion cascade (1). Critically, it is essential that leukocyte trafficking is tightly regulated as aberrant leukocyte recruitment into tissues contributes to the development of most immune-mediated chronic inflammatory diseases (IMIDs).

Leukocyte trafficking was first identified to be under circadian control over 50 years ago, when it was observed that circulating lymphocyte numbers oscillate according to the time of day (2). Since then, core circadian machinery has been identified in almost all immune cells and a strong reciprocal relationship between immunity and circadian clocks has been well established (3-5). Disruption of circadian rhythms due to genetic manipulation or lifestyle (for example, shift work) dysregulates the immune response and increases the susceptibility to cancers, cardiovascular disease, and metabolic disease (6). Several IMIDs show daily patterns of symptom intensity and 
responsiveness to treatment which has created a new avenue of chronotherapy that involves optimal timing of drug delivery (4). In older adults, leukocyte trafficking becomes dysregulated, contributing to age-related low-grade, chronic inflammation (inflammageing) that predispose the older population to IMIDs (7). Although it is known there is a dampening of circadian rhythms with increasing age, little work has been done to investigate the effects of this circadian disruption on the increase in IMIDs observed in older adults.

In this review, we explore the current knowledge regarding circadian control of leukocyte trafficking and the circadian oscillations of inflammatory conditions. We discuss the changes that occur to the circadian clock with increasing age and investigate whether this may contribute the age-related increase in inflammation and diseases.

\section{CIRCADIAN RHYTHM AND AGEING}

\section{The Master Pacemaker}

The circadian rhythm refers to the endogenous cycles seen in nearly all organisms that correlate with the Earth's 24-hour daynight cycle. Numerous biological processes are regulated by circadian clocks including behaviour, sleep, metabolism and body temperature $(8,9)$. In mammals, the master circadian pacemaker is found within the suprachiasmatic nucleus (SCN) which is entrained by the external environment and synchronises peripheral oscillators (10). Light enters the eye and sends input to the SCN via the retinohypothalamic tract enabling the central clock to entrain to external light/dark cues (11). SCN neurons send rhythmic outputs to peripheral organs and other brain areas, allowing global synchronisation with the external environment (12). Additionally, SCN neurons are able to generate autonomous circadian outputs allowing for circadian rhythms to exist even under constant darkness (13).

\section{Molecular Mechanisms}

In mammalian cells, the intracellular circadian clock is made up of an autoregulatory negative feedback loop. Transcriptional activators CLOCK (Circadian Locomotor Output Cycles Kaput) and BMAL1 (Brain and Muscle ARNT-Like 1) dimerise and form a complex. The CLOCK/BMAL1 complex then translocates to the nucleus where they bind to E-Box elements in promoter sequences of clock-controlled genes to positively regulate their own transcription (14). CLOCK/BMAL1 also promotes transcription of the clock regulators, Cryptochrome (CRY) and Period (PER), which in turn dimerise and undergo nuclear translocation where they inhibit CLOCK/BMAL1, repressing their own transcription (15). In addition to this feedback loop of core clock genes, nuclear receptor subfamilies Rev-erb and ROR (retinoic acid-related orphan receptor) compete for binding to ROR responsive elements (ROREs) in Bmal1 promoter sequences to repress and promote expression of BMAL1, respectively (16). Posttranslational modifications of clock transcription factors further regulate this feedback loop. Phosphorylation of PER and CRY proteins by casein kinase $\mathrm{I} \epsilon / \delta$ and AMP kinase promotes ubiquitination by E3 ligases resulting in their degradation (8).
Recently, another circadian repressor gene has been identified which is under control of the circadian clock (17). CHRONO (ChIP-derived repressor of network oscillator) inhibits CLOCK/ BMAL1 transcription activation in a histone deacetylase (HDAC)dependent manner, adding an epigenetic arm to the mammalian circadian clock (18). Overall, this negative feedback loop takes about 24 hours and results in the circadian oscillation seen in a multitude of physiological processes.

\section{Ageing and the Circadian Rhythm}

It is well-established that the circadian system influences ageing and longevity, and vice versa. Circadian outputs are diminished in older animals (19); transplantation of foetal SCN tissue into aged hamsters led to increases in longevity and restored the ageassociated loss of behavioural rhythmicity seen in control animals (20). BMAL1 knockout mice (KO) have significantly shorter lifespans than wild type (WT) controls, and display a premature ageing phenotype (21). Reactive oxygen species (ROS) accumulate in the kidney, spleen and heart of BMAL1 KO animals which all show an age-related decrease in size, suggesting a role of oxidative damage in age-associated degeneration. Inhibition of endogenous BMAL1 by siRNAs in murine fibroblast cell lines also increases ROS levels (6). Oxidative damage caused by increasing ROS production could drive the progression of cellular senescence of local cells, promoting a senescence-associated secretory phenotype, and subsequent dysregulation of the inflammatory response. CLOCK $\mathrm{KO}$ mice also have significantly reduced lifespans than WT controls, but show a milder ageing phenotype than BMAL1 KO mice, whereas PERIOD-deficient mice only have reduced lifespans after challenge with irradiation (22). The severe ageing phenotype limited to BMAL1 KO mice may be due to systemic effects independent of the circadian role of BMAL1, or functional redundancy seen by other core clock proteins. Importantly, BMAL1 KO mice also lose all time-of-day dependent leukocyte trafficking when housed in constant darkness, in contrast to WT littermates (23) indicating the pivotal role of the circadian clock in regulation of leukocyte trafficking.

In humans, ageing is associated with a reduced sleep quality and disrupted sleep cycles (9), which in turn further dysregulates the robustness of the circadian rhythm. Importantly, circadian rhythm dysregulation is associated with the development of agerelated disorders, including inflammatory and metabolic disorders, and neurodegenerative diseases such as Alzheimer's Disease and Parkinson's (24). Several changes occur to the circadian clock with increasing age and identifying which of these are natural processes of healthy ageing and which of these are pathological will increase our understanding of ageassociated aberrant inflammation.

\section{CIRCADIAN REGULATION OF LEUKOCYTE TRAFFICKING IN AGEING}

It is well established that leukocyte trafficking follows a circadian oscillation [reviewed extensively in $(3,4,25)$ ]. The expression of circadian clock genes is ubiquitous to nearly all immune cells, and clocks can directly regulate immune cell trafficking. 
Leukocyte trafficking becomes dysregulated in ageing as expression of adhesion molecules, chemokines and integrins change and senescent cells accumulate (26), contributing to inflammageing and increasing susceptibility to IMIDs. Despite the abundant research focusing on circadian changes with increasing age, very little work has been done on how this affects circadian control of leukocyte trafficking. Future research should concentrate on this interaction to identify which changes to the immune system and to the circadian machinery occur as a natural by-product of ageing and which are signs of pathology.

\section{Neutrophils}

Neutrophils are the first innate immune cell recruited to sites of inflammation, where they phagocytose pathogens and secrete antimicrobial agents (27). Under steady state conditions, neutrophils are retained in the bone marrow by the key retention signal, CXCL12, acting through its receptor, CXCR4. Diurnal adrenergic signals inhibit CXCL12 expression in the bone marrow, resulting in daily variations in chemokine expression which regulates the circadian egress of neutrophils (and haematopoietic stem cells) from the bone marrow $(28,29)$. Following LPS challenge, neutrophils show a circadian-regulated recruitment to the lungs (30). Interestingly, this is regulated by the circadian clocks in lung epithelial cells, and not the clocks within neutrophils themselves. Local lung epithelial cells regulate the diurnal expression of the chemokine, CXCL5, in a glucocorticoid-dependent mechanism, which attracts neutrophils to the lungs (30). This identifies the complexity of circadian regulation of leukocyte trafficking, as immune cells both contain intrinsic clock machinery and are regulated by chemokine expression, which can be under the control of circadian clocks in other cells.

Recently, it has been reported that human and murine neutrophils possess an intrinsic, cell-autonomous diurnal ageing programme that acts to regulate trafficking of neutrophils to infections, whilst promoting their removal from the bloodstream, thus protecting vessels from inflammation (31). In young (6-12 weeks old) WT mice, neutrophils lose CD62L expression and gain CXCR4 as they age, promoting their recruitment to the bone marrow for elimination (32). This diurnal change is mediated by BMAL1, which upregulates expression of CXCL2, enables autocrine surface CXCL2-CXCR2 interactions and in turn promotes CD62L expression (31). Neutrophil-specific CXCR4 KO mice showed constitutive ageing as seen by low levels of CD62L (31). Persistence of aged neutrophils in the vasculature of these mice increased thrombo-inflammation in a model of ischemiareperfusion, and depletion of these neutrophils prevented thrombus formation and improved survival after infarction (31). This suggests that the importance of this diurnal neutrophil ageing process is to prevent senescent neutrophils accumulating in the vasculature and to prevent thrombo-inflammation. Healthy aged mice have an accumulation of CD $11 b^{\text {high }} /$ ICAM- $1^{\text {high }}$ neutrophils in lymphoid organs (33) which may be in response to the increased levels of inflammation in aged mice. However, no research has been done on the diurnal neutrophil ageing process in aged mice and future work should aim to identify if this accumulation of neutrophils in aged lymphoid organs is a result of dysregulated neutrophil ageing due to reduced circadian outputs.

\section{Monocytes/Macrophages}

Macrophages are key regulators of the innate immune response and show strong circadian oscillations in genes involved in cytokine secretion, which are essential mediators of leukocyte trafficking (34). REV-ERB $\alpha$ has been highlighted as a direct link between the circadian clock and the macrophage inflammatory response (35) prompting further investigation of this nuclear receptor as a therapeutic target. The role of REV-ERB $\alpha$ in immune responses is well established [reviewed in (36)], and recently synthetic REV-ERB $\alpha$ agonists are being used in vivo to investigate a direct circadian modulation in IMIDs such as in Rheumatoid Arthritis (RA) and colitis. RA shows strong symptom rhythmicity, underpinned by daily fluctuations in serum IL-6 concentrations (37). Synthetic REV-ERB ligands have been shown to control the release of IL- 6 from macrophages and can alleviate disease symptoms (38). REVERBa also has a protective effect against colitis via downregulation of Nlrp3 inflammasome activity (39). Activation of REV-ERB $\alpha$ ameliorates ulcerative colitis in WT mice (39) suggesting it may be a promising target for colitis treatment.

Several aged-related changes occur in macrophages, including polarization towards an alternate M2 phenotype and reduction in phagocytosis (40). Despite M2 macrophages originally considered to display an 'anti-inflammatory' phenotype, these age-associated M2 like macrophages secrete several proinflammatory mediators including TNF $\alpha$, IL-1 and IL-6. In mice, Ly6C ${ }^{\text {high }}$ (inflammatory) monocytes but not Ly6C ${ }^{\text {low }}$ (patrolling) monocytes exhibit diurnal oscillations in trafficking under both homeostatic conditions and in a model of sterile peritonitis (41). A recent study discovered BMAL1 is induced following stimulation of M1, but not M2, macrophages by inflammatory stimuli (42). It appears that the classically activated, pro-inflammatory ' $\mathrm{M} 1$ ' phenotype is more tightly regulated by the circadian clock. Very little research has been done on the effect of ageing-associated circadian dysregulation and macrophage function, and it would be interesting to understand how the clock alters within ageing macrophages and whether circadian control of transcriptional programmes is affected. As discussed above, it appears that circadian regulation can have varying effects on different macrophage subsets, which highlights the complexity of the relationship between inflammation and circadian clocks. Targeting circadian mechanisms may be important to maintain homeostasis and responses to inflammation.

\section{Lymphocytes}

Numbers of T-cells in the circulation follow daily oscillations, with the highest numbers during the behavioural rest phase and decreasing up to $40 \%$ at the peak of the active phase (43). However, individual $\mathrm{T}$-cell subsets show varying migration patterns throughout the day, which is regulated by varying changes in serum concentrations of glucocorticoids and 
catecholamines (43). Cortisol levels peak in the blood at the beginning of the active phase and up-regulates IL-7 receptor (IL-7R) and the chemokine receptor, CXCR4, on the surface of naive and central memory T-cells, mediating their extravasation into the bone marrow. Conversely, numbers of circulating effector $\mathrm{CD}^{+} \mathrm{T}$-cells peak during the active phase at the same time as epinephrine. Effector $\mathrm{CD}^{+} \mathrm{T}$-cells have increased intrinsic expression of beta-2-adrenergic receptors and CXCR1, which is proposed to be the reason for the effector $\mathrm{CD} 8{ }^{+} \mathrm{T}$-cell response to epinephrine. This subset-specific variation in trafficking is thought to provide increased immune defence during the active phase, when injury or infection is most likely to occur $(43,44)$.

Numbers of lymphocytes in the lymph nodes fluctuate in the opposite manner than those in the circulation. In young WT mice, migration of lymphocytes to lymph nodes peaked at the start of the active phase roughly 8 hours after peak blood lymphocyte concentrations $(45,46)$. BMAL1 regulates rhythmic expression of CCR7 and the sphingosine-1-phosphate receptor (SIPR1) on lymphocytes, which mediates their homing to and egress from lymph nodes respectively (45). Lymphocyte egress from lymph nodes is also regulated by adrenergic innervation through B2adrenergic receptors (AR) (46). B2-AR-deficient mice lost the daily fluctuations of lymphocyte numbers in blood and lymph nodes due to reduced levels of norepinephrine in peripheral lymph nodes (46). It is thought that circadian oscillations in immune cells prime the immune system for stronger responses in the active phase when interaction with pathogens are most likely to occur. Retention of lymphocytes within the lymph nodes during the active phase is thought to increase the chance to encounter T-cells with their cognate antigen. These studies highlight the importance of both cell-intrinsic clocks and cell-extrinsic rhythmic signals for driving daily trafficking of lymphocytes.

T-cell recruitment is impaired in older adults, leading to a compromised adaptive immune response, increased vulnerability to infections, and weakened responses to vaccinations (reviewed in (26). Age-related dysregulation of lymphocyte recruitment has mostly been attributed to changes in expression of chemokines and adhesion molecules, and accumulation of senescent immune cells. However, very little research has investigated the contribution of diminished circadian outputs on lymphocyte trafficking in ageing.

\section{Endothelial Cells}

Leukocyte recruitment is also regulated by oscillating expression of adhesion molecules on the EC surface. Autonomic innervation via $\beta$-adrenoreceptors differentially regulates adhesion molecule expression in different tissues (23), resulting in a highly-tissue specific temporal expression of EC adhesion molecules. A screen of adhesion molecule expression in multiple murine organs revealed a general peak in expression of adhesion molecules on ECs at the start of the active phase, parallel to the increased leukocyte emigration from blood (47). Adoptive transfer of cells into EC-specific Bmal1-deficient mice lost the time-of-day dependent leukocyte migration out of the circulation seen in WT controls (47). Therefore, leukocyte recruitment is regulated by rhythmic expression of adhesion molecules on both the EC surface and the leukocyte surface, increasing efficacy of the leukocyte-endothelium interaction required for leukocyte rolling, adhesion, and transmigration across the endothelial barrier (Figure 1).

\section{CIRCADIAN RHYTHMS AND THE AGEING IMMUNE RESPONSE}

\section{Vaccination}

Older adults ( $>65$ years) often have weaker responses to primary vaccination than younger adults, in terms of titre and immunity to infection $(48,49)$. Older adults are particularly susceptible to infections and are at increased risk for serious complications due to ageing-related comorbidities and increased immunosenescence (50). Therefore, vaccine optimisation is essential to limit hospitalisation and deaths due to vaccine-preventable infections in the older population. Interestingly, Suzuki et al. (46) showed that in young (8-12 week) WT mice immunisation via intradermal injection of a soluble antigen conjugated with chicken $\gamma$-globulin (NP-CGG) resulted in an elevated humoral response when administered at peak lymph node lymphocyte cellularity (46). Recently, this has been confirmed in humans by administration of BCG vaccines. Early morning vaccination produced a stronger adaptive immune phenotype and increased cytokine production compared with later morning and evening administration (51). The immune microenvironment present during the initiation of an adaptive response is therefore an important regulator of the overall strength of the response, and timing of vaccine administration needs to be considered when developing and researching novel vaccines (52).

\section{Circadian Misalignment}

The importance of a robust circadian rhythm for maintaining health span with increasing age is evident as chronic circadian misalignment caused by night-shift work is associated with several age-related disorders (53). Adult, WT mice subjected to chronic jetlag by shifting light-dark conditions by an 8-hour phase advance every 4 days had significantly shorter lifespans than control mice, increased levels of senescent immune cells, and increased inflammatory cell infiltration to the liver indicating chronic inflammation (54). Another jet-lag model revealed significantly shorter lifespans of aged (27-31 months old), but not young (8-12 weeks old) C57BL/6 male mice, suggesting circadian misalignment has more severe consequences in aged animals (55). The exact mechanisms responsible for premature ageing seen in human shiftworkers are multifaceted and not fully understood.

A recent study found night-shift workers had increased plasma levels of C-reactive protein compared to day workers, indicating increased systemic inflammation (56). Importantly, night-workers had slightly decreased levels of long pentraxin 3 (PTX3), a pattern recognition receptor, which positively correlates with leukocyte telomere length, a marker of biological ageing. This suggests night-workers are more susceptible to premature ageing through increased systemic inflammation and loss of protective PTX3. Therefore, people 


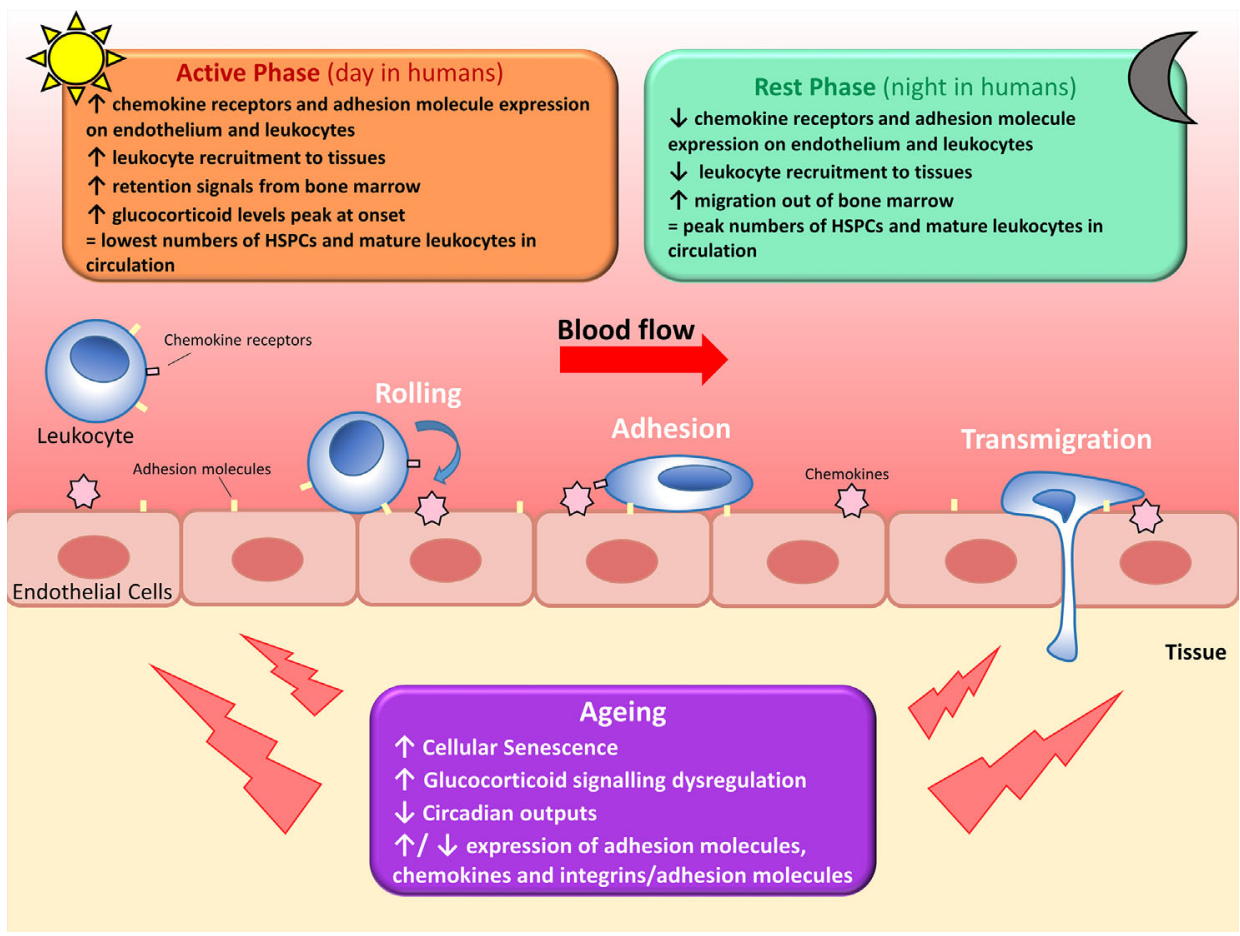

FIGURE 1 | Circadian regulation of leukocyte trafficking. Migration of leukocytes such as lymphocytes, neutrophils and monocytes, out of circulation and into surrounding tissues is regulated by circadian clocks. Hematopoietic stem cells (HSPCs) and mature leukocytes (except CD8 ${ }^{+} \mathrm{T}$ cells) peak in the circulation during the rest phase as there's less migration out of the blood and increased migration of leukocytes and haematopoietic stem cells out of the bone marrow. Conversely, circulating HPSCs and mature leukocytes (except CD8 ${ }^{+} \mathrm{T}$ cells) are at their lowest during the active phase due to increased leukocyte recruitment to tissues and reduced migration out of the bone marrow. Leukocyte migration is regulated by diurnal changes in expression of chemokines and adhesion molecules, and fluctuating glucocorticoid levels and adrenergic signalling. With increasing age, leukocyte trafficking becomes dysregulated due to a multitude of factors. Senescent cells accumulate and increase secretion of pro-inflammatory cytokines, glucocorticoid levels decrease and signalling becomes dysregulated, several age-associated changes occur to cytokine and adhesion molecule expression, and circadian outputs diminish. All of these contribute to dysregulated leukocyte trafficking seen in older adults.

who experience chronic circadian misalignment should consider the impact this may have on their health, and restoration of immune homeostasis may be a therapeutic target against agerelated disorders in these people.

\section{Inflammation and Rhythmicity: A Reciprocal Relationship}

Inflammation itself can directly affect circadian rhythmicity. TNF $\alpha$ inhibits the CLOCK/BMAL1-induced activation of E-box regulatory elements in clock-controlled genes in fibroblasts in vitro, and in livers of mice in vivo (5). Other recent studies in rheumatoid synovial cells have shown TNF $\alpha$ suppresses PER2, while inducing expression of BMAL1 by upregulating $\operatorname{ROR} \alpha$ (57). Additionally, long term treatment with IFN- $\gamma$ reduced the amplitude of the circadian rhythm of Per1-luc expression in individual cultured rat SCN neurons (58), and LPS injection caused transient suppression of core clock genes in male rats in vivo. These studies highlight the complex, reciprocal relationship between inflammation and clock genes, and support the idea increased inflammation seen in older adults may result in dysregulation of the circadian rhythm.

\section{FUTURE DIRECTIONS AND CONCLUSIONS}

Circadian rhythms play an essential role in immune homeostasis and regulate the diurnal rhythmicity seen in leukocyte trafficking under both steady state and inflammatory conditions. Inflammation itself can inhibit clock gene expression, demonstrating a complex and reciprocal relationship between the two biological systems. In older adults, there is a parallel increase in systemic inflammation and dysregulated leukocyte trafficking, and also a reduction of circadian outputs, both of which can enhance the other, therefore increasing vulnerability to disease. Despite a multitude of research into circadian systems and leukocyte trafficking, there is a need for more research into chronotherapy to optimise timing of drugs and vaccine delivery in order to improve drug efficacy, reduce side effects, and target chronic inflammation, particularly in aged individuals. Similarly, current work on vaccination has focussed on either how responses to vaccines vary with increasing age, or on how responses vary with the time of administration, but not on the two angles combined. Circadian rhythm research can also be 
beneficial to the development of non-pharmacological treatment strategies. For example, diminished circadian output leads to reduced sleep quality in older adults, which subsequently dysregulates global circadian rhythmicity. Entrainment of peripheral clocks via regulating food intake and light exposure may help alleviate the effects of dampened circadian outputs seen in older adults, and help prevent one of the contributing factors for increased inflammation. Importantly, ageing research struggles to characterise changes that occur as a natural result of healthy ageing, versus those that are a condition of ageassociated pathology, or changes in the circadian circuitry. More research into the circadian clock and inflammageing could determine if circadian rhythmicity can be used a sign of pathological ageing.

\section{REFERENCES}

1. Nourshargh S, Alon R. Leukocyte Migration Into Inflamed Tissues. Immunity (2014) 41:694-707. doi: 10.1016/j.immuni.2014.10.008

2. Elmadjian F, Pincus G. A Study of the Diurnal Variations in Circulating Lymphocytes in Normal and Psychotic Subjects. J Clin Endocrinol Metab (1946) 6(4):287-94. doi: 10.1210/jcem-6-4-287

3. Pick R, He W, Chen CS, Scheiermann C. Time-of-Day-Dependent Trafficking and Function of Leukocyte Subsets. Trends Immunol (2019) 40:524-37. doi: 10.1016/j.it.2019.03.010

4. Scheiermann C, Kunisaki Y, Frenette PS. Circadian Control of the Immune System. Nat Rev Immunol (2013) 13:190-8. doi: 10.1038/nri3386

5. Cavadini G, Petrzilka S, Kohler P, Jud C, Tobler I, Birchler T, et al. Tnf- $\alpha$ Suppresses the Expression of Clock Genes by Interfering With E-BoxMediated Transcription. Proc Natl Acad Sci USA (2007) 104(31):12843-8. doi: 10.1073/pnas.0701466104

6. Kondratov RV. A Role of the Circadian System and Circadian Proteins in Aging. Ageing Res Rev (2007) 6:12-27. doi: 10.1016/j.arr.2007.02.003

7. Franceschi C, Paolo G, Paolo P, Cristina G, Aurelia S. Inflammaging: a New Immune-Metabolic Viewpoint for Age-Related Diseases. Nat Rev Endocrinol (2018) 14:576-90. doi: 10.1038/s41574-018-0059-4

8. Mohawk JA, Green CB, Takahashi JS. Central and Peripheral Circadian Clocks in Mammals. Annu Rev Neurosci (2012) 35:445-62. doi: 10.1146/ annurev-neuro-060909-153128

9. Hood S, Amir S. The Aging Clock: Circadian Rhythms and Later Life. J Clin Invest (2017) 127:437-46. doi: 10.1172/JCI90328

10. Hastings MH, Maywood ES, Brancaccio M. Generation of Circadian Rhythms in the Suprachiasmatic Nucleus. Nat Rev Neurosci (2018) 19:453-69. doi: 10.1038/s41583-018-0026-z

11. Morin LP, Allen CN. The Circadian Visual System, 2005. Brain Res Rev (2006) 51:1-60. doi: 10.1016/j.brainresrev.2005.08.003

12. Yamazaki S, Numano R, Abe M, Hida A, Takahashi RI, Ueda M, et al. Resetting Central and Peripheral Circadian Oscillators in Transgenic Rats. Science (2000) 288(5466):682-5. doi: 10.1126/science.288.5466.682

13. Welsh DK, Logothetis DE, Meister M, Reppert SM. Dissociated From Rat Suprachiasmatic Nucleus Express Independently Phased Circadian Firing Rhythms. Neuron (1995) Apr 114(4):697-706. doi: 10.1016/0896-6273(95) 90214-7

14. Welsh DK, Takahashi JS, Kay SA. Suprachiasmatic Nucleus: Cell Autonomy and Network Properties. Annu Rev Physiol (2013) 72:551-7. doi: 10.1146/ annurev-physiol-021909-135919

15. Huang W, Ramsey KM, Marcheva B, Bass J. Circadian Rhythms, Sleep, and Metabolism. J Clin Invest (2011) 121:2133-41. doi: 10.1172/JCI46043

16. Guillaumond F, Dardente H, Giguère V, Cermakian N. Differential Control of Bmall Circadian Transcription by REV-ERB and ROR Nuclear Receptors. J Biol Rhythms (2005) 20(5):391-403. doi: 10.1177/0748730405277232

17. Goriki A, Hatanaka F, Myung J, Kim JK, Yoritaka T, Tanoue S, et al. Novel Protein, CHRONO, Functions as a Core Component of the Mammalian

\section{AUTHOR CONTRIBUTIONS}

PN wrote the first draft of the manuscript. MC, JG, and GR contributed to manuscript revision, read and approved the submitted version.

\section{FUNDING}

PN was supported by a Wellcome Trust PhD studentship (108871/ Z/15/Z) and MC was supported by a Royal Society Dorothy Hodgkin Research fellowship (DH160044). JG is supported by the MRC (MR/S002715/1 and MR/P023576/1). GR is supported by the British Heart Foundation (FS/20/2/34799).

Circadian Clock. PloS Biol (2014) 12(4):1001839. doi: 10.1371/ journal.pbio.1001839

18. Yang Y, Li N, Qiu J, Ge H, Qin X. Identification of the Repressive Domain of the Negative Circadian Clock Component CHRONO. Int J Mol Sci (2020) 21 (7):2469. doi: 10.3390/ijms21072469

19. Nakamura TJ, Nakamura W, Yamazaki S, Kudo T, Cutler T, Colwell CS, et al. Age-Related Decline in Circadian Output. J Neurosci (2011) 31(28):10201-5. doi: 10.1523/JNEUROSCI.0451-11.2011

20. Hurd MW, Ralph MR. The Significance of Circadian Organization for Longevity in the Golden Hamster. J Biol Rhythms (1998) 13(5):430-6. doi: $10.1177 / 074873098129000255$

21. Kondratov RV, Kondratova AA, Gorbacheva VY, Vykhovanets OV, Antoch MP. Early Aging and Age-Related Pathologies in Mice Deficient in BMAL1, the Core Component of the Circadian Clock. Genes Dev (2006) 20(14):186873. doi: $10.1101 /$ gad.1432206

22. Dubrovsky YV, Samsa WE, Kondratov RV. Deficiency of Circadian Protein CLOCK Reduces Lifespan and Increases Age-Related Cataract Development in Mice. Aging (Albany NY) (2010) 2(12):936-44. doi: 10.18632/aging.100241

23. Scheiermann C, Kunisaki Y, Lucas D, Chow A, Jang JE, Zhang D, et al. Adrenergic Nerves Govern Circadian Leukocyte Recruitment to Tissues. Immunity (2012) 37(2):290-301. doi: 10.1016/j.immuni.2012.05.021

24. Mattis J, Sehgal A. Circadian Rhythms, Sleep, and Disorders of Aging. Trends Endocrinol Metab (2016) 27:192-203. doi: 10.1016/j.tem.2016.02.003

25. Yuan Y, Wu S, Li W, He W. A Tissue-Specific Rhythmic Recruitment Pattern of Leukocyte Subsets. Front Immunol (2020) 11:102/full. doi: 10.3389/ fimmu.2020.00102/full

26. Hopkin S, Lord JM, Chimen M. Dysregulation of Leukocyte Trafficking in Ageing: Causal Factors and Possible Corrective Therapies. Pharmacol Res (2020) 163. doi: 10.1016/j.phrs.2020.105323

27. Rosales C. Neutrophil: a Cell With Many Roles in Inflammation or Several Cell Types? Front Physiol (2018) 9:113. doi: 10.3389/fphys.2018.00113

28. Méndez-Ferrer S, Lucas D, Battista M, Frenette PS. Haematopoietic Stem Cell Release is Regulated by Circadian Oscillations. Nature (2008) 452(7186):4427. doi: 10.1038/nature06685

29. Aroca-Crevillén A, Adrover JM, Hidalgo A. Circadian Features of Neutrophil Biology. Front Immunol (2020) 11:576. doi: 10.3389/fimmu.2020.00576

30. Gibbs J, Ince L, Matthews L, Mei J, Bell T, Yang N, et al. An Epithelial Circadian Clock Controls Pulmonary Inflammation and Glucocorticoid Action. Nat Med (2014) 20(8):919-26. doi: 10.1038/nm.3599

31. Adrover JM, del Fresno C, Crainiciuc G, Cuartero MI, Casanova-Acebes M, Weiss LA, et al. A Neutrophil Timer Coordinates Immune Defense and Vascular Protection. Immunity (2019) 50(2):390-402.e10. doi: 10.1016/ j.immuni.2019.01.002

32. Casanova-Acebes M, Pitaval C, Weiss LA, Nombela-Arrieta C, Chèvre R, AGonzález N, et al. Rhythmic Modulation of the Hematopoietic Niche Through Neutrophil Clearance. Cell (2013) 153(5):1025. doi: 10.1016/j.cell.2013.04.040

33. Tomay F, Wells K, Duong L, Tsu JW, Dye DE, Radley-Crabb HG, et al. Aged Neutrophils Accumulate in Lymphoid Tissues From Healthy Elderly Mice 
and Infiltrate T-and B-Cell Zones. Immunol Cell Biol (2018) 96(8):831-40. doi: $10.1111 /$ imcb. 12046

34. Keller M, Mazuch J, Abraham U, Eom GD, Herzog ED, Volk HD, et al. A Circadian Clock in Macrophages Controls Inflammatory Immune Responses. Proc Natl Acad Sci USA (2009) 106(50):21407-12. doi: 10.1073/ pnas. 0906361106

35. Gibbs JE, Blaikley J, Beesley S, Matthews L, Simpson KD, Boyce SH, et al. The Nuclear Receptor REV-Erb $\alpha$ Mediates Circadian Regulation of Innate Immunity Through Selective Regulation of Inflammatory Cytokines. Proc Natl Acad Sci USA (2012) 109(2):582-7. doi: 10.1073/pnas.1106750109

36. Hergenhan S, Holtkamp S, Scheiermann C. Molecular Interactions Between Components of the Circadian Clock and the Immune System. J Mol Biol (2020) 432:3700-13. doi: 10.1016/j.jmb.2019.12.044

37. Hand LE, Hopwood TW, Dickson SH, Walker AL, Loudon ASI, Ray DW, et al. The Circadian Clock Regulates Inflammatory Arthritis. FASEB J (2016) 30(11):3759-70. doi: 10.1096/fj.201600353R

38. Liu H, Zhu Y, Gao Y, Qi D, Zhao L, Zhao L, et al. NR1D1 Modulates Synovial Inflammation and Bone Destruction in Rheumatoid Arthritis. Cell Death Dis (2020) 11(2):129. doi: 10.1038/s41419-020-2314-6

39. Wang S, Lin Y, Yuan X, Li F, Guo L, Wu B. Rev-Erb $\alpha$ Integrates Colon Clock With Experimental Colitis Through Regulation of NF-kb/NLRP3 Axis. Nat Commun (2018) 9(1):4246. doi: 10.1038/s41467-018-06568-5

40. van Beek AA, Van den Bossche J, Mastroberardino PG, de Winther MPJ, Leenen PJM. Metabolic Alterations in Aging Macrophages: Ingredients for Inflammaging? Trends Immunol (2019) 40:113-27. doi: 10.1016/ j.it.2018.12.007

41. Nguyen KD, Fentress SJ, Qiu Y, Yun K, Cox JS, Chawla A. Gene Bmal1 Regulates Diurnal Oscillations of Ly6Chi Inflammatory Monocytes. Science (2013) 341(6153):1483-8. doi: 10.1126/science.1240636

42. Alexander RK, Liou YH, Knudsen NH, Starost KA, Xu C, Hyde AL, et al. Bmal1 Integrates Mitochondrial Metabolism and Macrophage Activation. Elife (2020) 9:e54090. doi: 10.7554/eLife.54090

43. Dimitrov S, Benedict C, Heutling D, Westermann J, Born J, Lange T. Cortisol and Epinephrine Control Opposing Circadian Rhythms in T Cell Subsets. Blood (2009) 113(21):5134-43. doi: 10.1182/blood-2008-11-190769

44. Lange T, Besedovsky L, Schmidt E, Born J. 75. Circadian Rhythms of Human T Cell Subsets. Brain Behav Immun (2013) 32:e22. doi: 10.1016/ j.bbi.2013.07.087

45. Druzd D, Matveeva O, Ince L, Harrison U, He W, Schmal C, et al. Lymphocyte Circadian Clocks Control Lymph Node Trafficking and Adaptive Immune Responses. Immunity (2017) 46(1):120-32. doi: 10.1016/j.immuni.2016.12.011

46. Suzuki K, Hayano Y, Nakai A, Furuta F, Noda M. Adrenergic Control of the Adaptive Immune Response by Diurnal Lymphocyte Recirculation Through Lymph Nodes. J Exp Med (2016) 213(12):2567-74. doi: 10.1084/jem.20160723

47. He W, Holtkamp S, Hergenhan SM, Kraus K, de Juan A, Weber J, et al. Circadian Expression of Migratory Factors Establishes Lineage-Specific Signatures that Guide the Homing of Leukocyte Subsets to Tissues. Immunity (2018) 196(6):1175-90.e7. doi: 10.1016/j.immuni.2018.10.007

48. Wagner A, Garner-Spitzer E, Jasinska J, Kollaritsch H, Stiasny K, Kundi M, et al. Age-Related Differences in Humoral and Cellular Immune Responses
After Primary Immunisation: Indications for Stratified Vaccination Schedules. Sci Rep (2018) 8(1):9825. doi: 10.1038/s41598-018-28111-8

49. Murasko DM, Bernstein ED, Gardner EM, Gross P, Munk G, Dran S, et al. Role of Humoral and Cell-Mediated Immunity in Protection From Influenza Disease After Immunization of Healthy Elderly. Exp Gerontol (2002) 37(23):427-39. doi: 10.1016/S0531-5565(01)00210-8

50. Lord JM. The Effect of Aging of the Immune System on Vaccination Responses. Hum Vaccines Immunother (2013) 9:1364-7. doi: 10.4161/ hv. 24696

51. Charlotte L, Mourits VP, Koeken VACM, Moorlag SJCFM, Janssen R, Folkman L, et al. Circadian Rhythm Influences Induction of Trained Immunity by BCG Vaccination. J Clin Invest (2020) 130(10):5603-17. doi: 10.1172/JCI133934

52. Green KN, Billings LM, Roozendaal B, McGaugh JL, LaFerla FM. Glucocorticoids Increase Amyloid- $\beta$ and Tau Pathology in a Mouse Model of Alzheimer's Disease. J Neurosci (2006) 26(35):9047-56. doi: 10.1523/ JNEUROSCI.2797-06.2006

53. Cuesta M, Boudreau P, Dubeau-Laramée G, Cermakian N, Boivin DB. Simulated Night Shift Disrupts Circadian Rhythms of Immune Functions in Humans. J Immunol (2016) 196(6):2466-75. doi: 10.4049/jimmunol.1502422

54. Inokawa H, Umemura Y, Shimba A, Kawakami E, Koike N, Tsuchiya Y, et al. Chronic Circadian Misalignment Accelerates Immune Senescence and Abbreviates Lifespan in Mice. Sci Rep (2020) 10(1):1-13. doi: 10.1038/ s41598-020-59541-y

55. Davidson AJ, Sellix MT, Daniel J, Yamazaki S, Menaker M, Block GD. Chronic Jet-Lag Increases Mortality in Aged Mice. Curr Biol (2006) 16: R914-6. doi: 10.1016/j.cub.2006.09.058

56. Pavanello S, Stendardo M, Mastrangelo G, Bonci M, Bottazzi B, Campisi M, et al. Inflammatory Long Pentraxin 3 is Associated With Leukocyte Telomere Length in Night-Shift Workers. Front Immunol (2017) 8(MAY):1. doi: 10.3389/fimmu.2017.00516

57. Yoshida K, Nakai A, Kaneshiro K, Hashimoto N, Suzuki K, Uchida K, et al. Tnf- $\alpha$ Induces Expression of the Circadian Clock Gene Bmall Via Dual Calcium-Dependent Pathways in Rheumatoid Synovial Cells. Biochem Biophys Res Commun (2018) 495(2):1675-80. doi: 10.1016/j.bbrc.2017.12.015

58. Kwak Y, Lundkvist GB, Brask J, Davidson A, Menaker M, Kristensson K, et al. Interferon- $\gamma$ Alters Electrical Activity and Clock Gene Expression in Suprachiasmatic Nucleus Neurons. J Biol Rhythms (2008) 23(2):150-9. doi: $10.1177 / 0748730407313355$

Conflict of Interest: The authors declare that the research was conducted in the absence of any commercial or financial relationships that could be construed as a potential conflict of interest.

Copyright $\odot 2021$ Nathan, Gibbs, Rainger and Chimen. This is an open-access article distributed under the terms of the Creative Commons Attribution License (CC BY). The use, distribution or reproduction in other forums is permitted, provided the original author(s) and the copyright owner(s) are credited and that the original publication in this journal is cited, in accordance with accepted academic practice. No use, distribution or reproduction is permitted which does not comply with these terms. 